\title{
Quantum dot-based fluorescent probes for targeted imaging of the EJ human bladder urothelial cancer cell line
}

\author{
RUN YUAN $^{1 *}$, TING RAO $^{1 *}$, FAN CHENG $^{1}$, WEI-MIN YU ${ }^{1}$, \\ YUAN RUAN $^{1}$, XIAO-BIN ZHANG ${ }^{1}$ and STÉPHANE LARRÉ ${ }^{2}$ \\ ${ }^{1}$ Department of Urology, Renmin Hospital of Wuhan University, Wuhan, Hubei 430060, P.R. China; \\ ${ }^{2}$ Department of Urology, Robert Debré Teaching Hospital, University of Reims, 51100 Reims, France
}

Received February 11, 2017; Accepted March 16, 2018

DOI: $10.3892 / \mathrm{etm} .2018 .6805$

\begin{abstract}
QDs are a type of inorganic nanoparticle with unique optical properties. As a fluorescent label, QDs are widely used in biomedical fields. In the present study, fluorescent probes of quantum dots (QDs) conjugated with a prostate stem cell antigen (PSCA) monoclonal antibody (QD-PSCA) were prepared to study the targeted imaging of QD-PSCA probes in EJ human bladder urothelial cancer cells and analyze the feasibility of QD-based non-invasive tumor-targeted imaging in vivo. QDs with an emission wavelength of $605 \mathrm{~nm}$ (QD605) were conjugated with PSCA to prepare QD605-PSCA fluorescent probes by chemical covalent coupling. The optical properties of the probes coupled and uncoupled with PSCA were measured and assessed using an ultraviolet spectrophotometer and a fluorescence spectrophotometer. Direct immune-fluorescent labeling was utilized to detect and analyze imaging of the probes for EJ cells. The results revealed that QD605-PSCA probes retained the fluorescent properties of QD605 and the immunogenicity of the PSCA protein. The probes were able to specifically recognize the PSCA protein expressed in bladder cancer cells, while fluorescence was stable and had a long duration. The present study suggests that QD-PSCA fluorescent probes may be useful for specific targeted labeling and imaging in bladder urothelial cancer cells. Furthermore, the probes possess good optical stability and may be useful for research into non-invasive targeted imaging, early diagnosis and targeted in vivo tumor therapy.
\end{abstract}

\section{Introduction}

Bladder cancer is the ninth most common malignant tumor in the world (1). In 2015, there were 74,000 newly diagnosed cases and $\sim 16,000$ bladder cancer-associated mortalities in the USA (2). In

Correspondence to: Dr Fan Cheng, Department of Urology, Renmin Hospital of Wuhan University, 99 Zhangzhidong Road, Wuhan, Hubei 430060, P.R. China

E-mail: urology1969@aliyun.com

${ }^{*}$ Contributed equally

Key words: bladder cancer, quantum dots, PSCA, fluorescent probes
China, 80,500 cases of bladder cancer were newly diagnosed and $\sim 32,900$ people succumbed to the disease in 2015 (3). Over $90 \%$ of cases are bladder urothelial cancer (BUC) and the typical biological behavior characteristics of BUC include recurrence and progression (4). Enabling the early detection of tumor recurrence and metastasis is a major problem faced by researchers and is of great significance for timely and appropriate clinical treatment and judging tumor prognosis. Therefore, feasible in vivo non-invasive visualization of tumors would be advantageous for early diagnosis and monitoring of BUC.

Quantum dots (QDs) are a type of inorganic nanoparticle with unique optical properties. Compared with traditional organic fluorescent probes, QDs possess wide absorption spectra, a narrow emission peak, high fluorescence intensity, long fluorescent duration, stable long-term fluorescence and strong resistance to photobleaching (5-8). Due to their optical properties, QDs have a broad range of potential applications in molecular and cellular labeling, imaging and tracking in vitro and in vivo.

Prostate stem cell antigen (PSCA) is a type of prostate tumor-associated antigen, which is homologous to the cell surface antigen of the Thy-1/Ly-6 family (9). In normal prostate and bladder epithelium, the expression of PSCA is low; however, PSCA is overexpressed in prostate, kidney and bladder cancer (9-11).

In the present study, QD fluorescent probes with an emission wavelength of $605 \mathrm{~nm}$ (QD605) conjugated to the PSCA monoclonal antibody (QD605-PSCA) by covalent coupling were prepared. QD-fluorescence labeling was used to observe the specific imaging of the QD605-PSCA probes in the EJ human bladder urothelial cancer cell line. The results were used to analyze the feasibility of in vivo non-invasive targeted imaging of the probes in bladder cancer.

\section{Materials and methods}

Materials and instruments. The human bladder urothelial carcinoma cell line EJ was a gift from the Urology Department, Zhongnan Hospital of Wuhan University (Wuhan, China), which is known to be cross-contaminated with the T24 bladder carcinoma cell line $(12,13)$. Fetal bovine serum (FBS), RPMI-1640 and $0.25 \%$ trypsin were purchased from Hyclone (GE Healthcare Life Sciences, Logan, UT, USA). The mouse anti-human monoclonal antibody PSCA (cat. no. ab56338) was purchased 
from Abcam (Cambridge, MA, USA). The QD605 Antibody Conjugation kit was purchased from Qingdao Jiayuan Group Co., Ltd. (Wuhan, China). Succinimidyl-4-(N-maleimidomethyl) cyclohexane-1-carboxylate (SMCC), dithiothreitol (DTT) and 2-(N-Morpholino) ethanesulfonic Acid (MES) were purchased from Thermo Fisher Scientific, Inc. (Waltham, MA, USA). The fluorescence microscope and inverted fluorescence microscope were purchased from Olympus Corp. (Tokyo, Japan). The fluorescence spectrophotometer was from PerkinElmer, Inc., (Waltham, MA, USA). The ultraviolet spectrophotometer was from Shimadzu (Kyoto, Japan).

Cell culture. EJ cells were cultivated in RPMI-1640 medium containing $10 \%$ FBS and $1 \%$ penicillin-streptomycin in an incubator with humidified air under an atmosphere of $5 \% \mathrm{CO}_{2}$ and a temperature of $37^{\circ} \mathrm{C}$. When the cells reached at least $80 \%$ confluence, they were subcultured. EJ cells were maintained at $37^{\circ} \mathrm{C}$ with in an atmosphere containing $5 \% \mathrm{CO}_{2}$.

Preparation of QD605-PSCA probes. QD605-PSCA antibody probes were created using the QD605 antibody conjugation kit according to the manufacturer's protocol (all reagents and equipment mentioned subsequently were included in this kit). The first step involved the activation and purification of QDs. A total of $100 \mu 1$ of amino QD605 (amine-functionalized CdSe/ZnS) at a concentration of $8 \mu \mathrm{M}$ was thoroughly mixed with $11 \mu \mathrm{l}$ SMCC at a concentration of $10 \mathrm{mM}$ in a $2 \mathrm{ml}$ centrifuge tube. Following $1 \mathrm{~h}$ of shaking at room temperature, the reaction liquid was transferred to a desalination column. The colored elution was then collected with the MES elution buffer. The second step involved the antibody reduction and purification. A total of $300 \mu \mathrm{l}$ of the PSCA monoclonal antibody $(1 \mathrm{mg} / \mathrm{ml})$ was thoroughly mixed with $6.1 \mu 1$ of DTT $(1 \mathrm{M})$ in a $2 \mathrm{ml}$ centrifuge tube. Following $30 \mathrm{~min}$ of the reduction reaction, the colored solution was collected by elution from the desalination column. The next steps involved conjugation and purification. The elution solutions collected in the first two steps were mixed for $1 \mathrm{~h}$ in the conjugation reaction. Then, according to different molecular sizes, molecular sieve column chromatography and ultrafiltration were used to separate the unreacted antibody. Finally, the QD605-PSCA probes were obtained.

Detection of the optical properties of QD605-PSCA probes. The absorption and emission spectra of QD605 and QD605-PSCA were measured with the ultraviolet spectrophotometer and fluorescence spectrophotometer, respectively. The UV scanning wavelength range was 400 to $700 \mathrm{~nm}$ with a $0.5 \mathrm{~nm}$ scanning interval. The fluorescence excitation wavelength was $380 \mathrm{~nm}$ with a $1 \mathrm{~nm}$ scan step. The reference solution was $10 \mathrm{mM}$ PBS with a $\mathrm{pH}$ of 7.2.

Specific fluorescent labeling of EJ fixed cells with QD605-PSCA probes. EJ cells in the logarithmic phase were trypsinized and resuspended in RPMI 1640 medium. A $0.1 \mathrm{ml}$ cell resuspension $\left(3 \times 10^{5}\right.$ cells) was inoculated onto a 6 -well culture plate and cells were cultivated in an incubator at $37^{\circ} \mathrm{C}$ with $5 \% \mathrm{CO}_{2}$. When the growth density was $50-70 \%$, the cover glass was removed, EJ cells were fixed by adding an ice-cold acetone solution to cover each cover glass and the acetone underwent natural drying. The slides were stored at $-20^{\circ} \mathrm{C}$.
The slides containing the EJ fixed cells were washed lightly with TBS twice, incubated at $37^{\circ} \mathrm{C}$ with $0.1 \%$ Triton-X 100 for $10 \mathrm{~min}$, washed twice with double distilled $\mathrm{H}_{2} \mathrm{O}$, washed twice with TBS and blocked with $2 \%$ bovine serum albumin purchased form Wuhan Jiayuan Quantum Dot Technological Development Co., Ltd. (Wuhan, China) for $30 \mathrm{~min}$ at $37^{\circ} \mathrm{C}$. Each cover glass was divided into two parts and the cells on each cover glass were divided into three groups. $0.05 \mathrm{ml}$ of the QD605-PSCA probe solution that was diluted with TBS (1:50) was added the surface of the cover glass in the experimental group. The labeling solution was replaced with an equal volume of free QD605 or TBS in the control groups I and II, respectively. Each group was set up in triplicate. The cells were incubated at $4^{\circ} \mathrm{C}$ overnight. The labeling solution was washed off and cells were lightly washed with TBS-T and TBS three times to remove the unbound QD605-PSCA and QD605. A total of $0.05 \mathrm{ml}$ DAPI solution was then diluted with TBS (1:300) and added to the surface of the cover glass in all groups for $5 \mathrm{~min}$ at $37^{\circ} \mathrm{C}$ and cells were lightly washed with TBS three times to remove unbound DAPI. Finally, slides were placed on a glass slide with neutral glycerin and a fluorescence microscope was used to observe the labeling (magnification, $\mathrm{x} 400$ ).

Fluorescent labeling of EJ living cells with QD605-PSCA probes. Exponentially growing EJ cells $\left(1 \times 10^{5}\right.$ cells/well) were inoculated onto a 12-well plate. These cells were cultivated in an incubator at $37^{\circ} \mathrm{C}$ with $5 \% \mathrm{CO}_{2}$. When the growth density was $\sim 70 \%$, the fluorescent labeling experiment of living cells was initiated. The key steps were as follows: The original culture medium was discarded and cells were lightly washed with PBS three times. Cells were divided into three groups. $0.1 \mathrm{ml}$ of the QD605-PSCA probe solution (10 nM) diluted with PBS (1:50) was added to each well in the experimental group. The labeling solution was replaced with an equal volume of free QD605 or PBS in control groups I and II, respectively. Each group had three equal wells. The cells were incubated in an incubator at $37^{\circ} \mathrm{C}$ with $5 \% \mathrm{CO}_{2}$ for $30 \mathrm{~min}$. Then, the labeling solution was discarded and the cells were lightly washed with PBS three times to remove unbound QD605-PSCA or QD605. An inverted fluorescence microscope was used to observe the labeling. After capturing images of the cells, RPMI 1640 medium was added to the experimental group for continued culturing and EJ living cells were observed with the inverted fluorescence microscope at 6,24 and $48 \mathrm{~h}$ (magnification, x200).

Statistical analysis. All statistical analyses were performed using SPSS 17.0 (SPSS, Inc., Chicago, IL, USA). The statistical differences between the groups were analyzed using the Rank sum test. $\mathrm{P}<0.05$ was considered to indicate a statistically significant difference.

\section{Results}

Preparation of QD605-PSCA probes. The coupling products were analyzed using Ready Pouch ${ }^{\mathrm{TM}}$ Agerose Gels which was from Invitrogen (Carlsbad, California, USA) The migration velocity is associated with the surface charge, with more negative charges resulting in a faster speed of moving to the anode (14). In the activated reaction, the cationic amino group of QD605 was bound to the carboxyl group on SMCC, which neutralized the 
positive charges of QD605. In addition, SMCC was negatively charged in water solutions due to the anionic sulfonic group. Therefore, the migration speed of QD605-SMCC was faster than QD605. The thiol group on reductive PSCA was connected with the maleimide group on the intermediate of QD605-SMCC to produce the QD605-PSCA complex, increasing the molecular weight and resulting in a slower migration speed for QD605-PSCA compared with QD605-SMCC (Fig. 1).

Optical properties of QD605-PSCA probes. The absorption and emission spectra of the QD605 and QD605-PSCA probes were determined using the ultraviolet spectrophotometer and fluorescence spectrophotometer, respectively. Following QD605 coupling with PSCA, the absorption spectrum of the QD605-PSCA probes was wide and continuous. The absorbance spectrum of QD605-PSCA was higher compared with that of QD605 ( $<<0.05$; Table I; Fig. 2A). The emission spectrum of QD605-PSCA was narrow and symmetrical with an obvious fluorescence emission peak at $\sim 608 \mathrm{~nm}$. No significant difference was observed between the fluorescence intensities of QD605-PSCA and QD605 (Table I; Fig. 2).

Specific fluorescent labeling of EJ fixed cells with QD605PSCA probes. The fluorescence of EJ cells labeled with QD605-PSCA was examined using fluorescence microscopy. Orange-red fluorescence of the QD605-PSCA probes was observed in the cell membrane and cytoplasm, whereas the cell nucleus fluoresced blue following DAPI staining (Fig. 3A). In the control group, QD605 was not conjugated with the PSCA antibody. Due to repeated washing, nonspecific adsorption of QDs and unbound QDs was removed and no obvious orange-red fluorescence was observed (Fig. 3B). Labeled slides were stored at $4^{\circ} \mathrm{C}$ and fluorescence was observed every $24 \mathrm{~h}$. The blue fluorescence of the nuclei disappeared following $48 \mathrm{~h}$, whereas orange-red fluorescence of the QD605-PSCA probe signal was still visible on the seventh day (data not shown).

Fluorescent labeling of EJ living cells with QD605-PSCA probes. The fluorescence of EJ living cells labeled with the QD605-PSCA probes was observed using an inverted fluorescence microscope (Fig. 4). The orange-red fluorescence of the QD605-PSCA probes was observed in the cell membrane (Fig. 4A). In the control group, QD605 was not conjugated with PSCA and QD605 was not labeled with EJ cells directly; as such, the fluorescence was consistent with the background (Fig. 4D). Over time, the QD605-PSCA probes were taken up into the cytoplasm. Due to the influence of cell division and the culture medium, the average fluorescence intensity was weakened gradually but remained visible after $48 \mathrm{~h}$ (Fig. 4B and C).

\section{Discussion}

In recent years, with the development of synthesis technology and surface chemical modification, the toxicity, biological compatibility and biological molecular coupling of QDs have been effectively improved (15). QD-based fluorescent probes are widely used in chemistry, biology, medicine and other fields due to their favorable optical properties and biological compatibility $(16,17)$. Studies have revealed that QDs are the most ideal fluorescent probes for surface receptor imaging in cellular

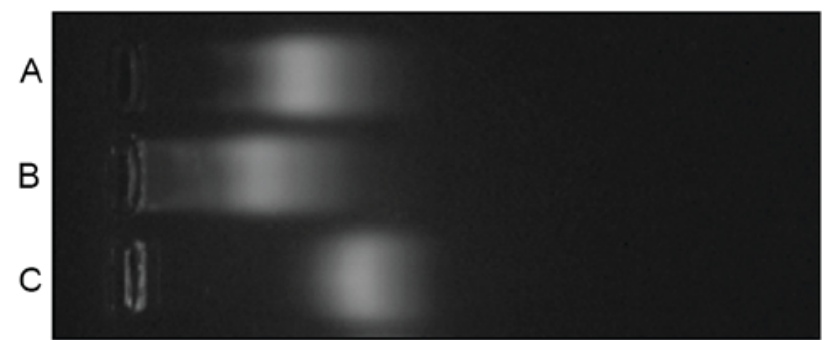

Figure 1. Agarose gel electrophoresis of (A) QD605-PSCA, (B) QD605 and (C) QD605-SMCC. The results demonstrated that the migration speed of QD605-SMCC was faster than QD605 and the QD605-PSCA complex. However, the speed of QD605-PSCA complex was faster than QD605. QD, quantum dot; PSCA, prostate stem cell antigen; SMCC, succinimidyl-4-(N-maleimidomethyl) cyclohexane-1-carboxylate.

labeling (18-20). In imaging studies, QDs are able to produce biological effects through modifying antibodies, peptides, proteins, nucleic acids or other biological molecules (21-23).

QD coupling with biological molecules is typically divided into non-covalent and covalent coupling. Non-covalent coupling primarily includes electrostatic interactions and the avidin-biotin complex method. Electrostatic interactions are a type of early biological coupling method, where the positively charged proteins are connected with the negatively charged QDs by electrostatic attraction (24). This method is quite simple, but vulnerable to the effects of $\mathrm{pH}$ and ionic strength in biological environment (24). The avidin-biotin method is one of the most widely applied biological coupling technologies, which exhibits high selectivity and strong bonding (25). However, this method usually causes an aggregated reaction (24-26). The combination of the two factors may increase the molecular weight of the product with worse tissue permeability, which would reduce the labeling efficiency (27).

The reaction between carboxyl and amino vs. maleic imide and sulfur is commonly used for covalent coupling. The former typically comprises the coupling of carboxyl on the QDs with the amino on the molecules via an amide bond based on the EDC [1-ethyl-3-(3-dimethylaminopropyl) carbodiimide]/NHS (N-hydroxy-succinamide) (28). The carboxyl and amino functional groups are rich in biological molecules and can be easily introduced into the surface of the QDs (28-30). However, during the binding reaction, EDC and its intermediates will rapidly hydrolyze in the aqueous medium and the total efficiency of the binding reaction is not high (28). In addition, most peptides and proteins containing a free carboxyl acid and/or amino acid are not easy to use in the binding reaction due to unnecessary cross-reaction between the constituents (28). The covalent bonding of maleic imide and thiol depends on the formation of the thioether bond. The binding reaction has high efficiency and strong specificity neutral $\mathrm{pH}$ conditions (28). The commercially available maleic imide reagent can be directly combined to be exposed to a certain amino acid group in the QDs, which enables these QDs to couple with biological molecules, including thiol groups (28).

In the present study, the covalent binding of QD605 and the antibody was based on an SMCC coupling agent (31). SMCC is a type of cross-linking agent with double functional groups that can activate the amino groups of the QD605 to produce the QD605-SMCC polymer (31). In addition, under 
Table I. Absorbance and fluorescence intensity of QD605 and QD605-PSCA probes.

\begin{tabular}{lccc}
\hline Group & Number & Absorbance (mean rank) & Fluorescence intensity (mean rank) \\
\hline QD605 & 601 & 562.43 & 596.36 \\
QD605-PSCA & 601 & 640.57 & $606.64^{\mathrm{a}}$ \\
\hline
\end{tabular}

${ }^{a} \mathrm{P}>0.05$ vs. QD605 group. QD, quantum dot; PSCA, prostate stem cell antigen.

A

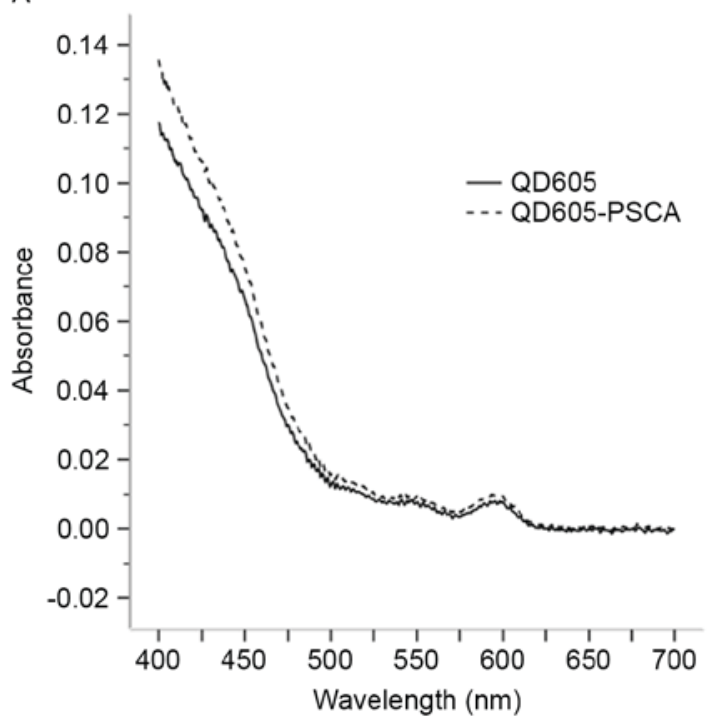

B

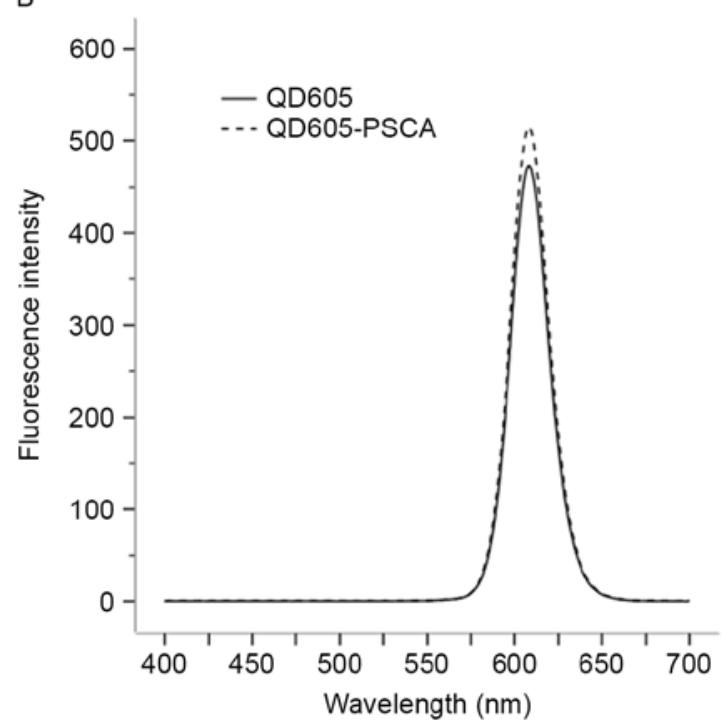

Figure 2. (A) Absorption and (B) emission spectra of QD605 and QD605-PSCA probes. QD, quantum dot; PSCA, prostate stem cell antigen.
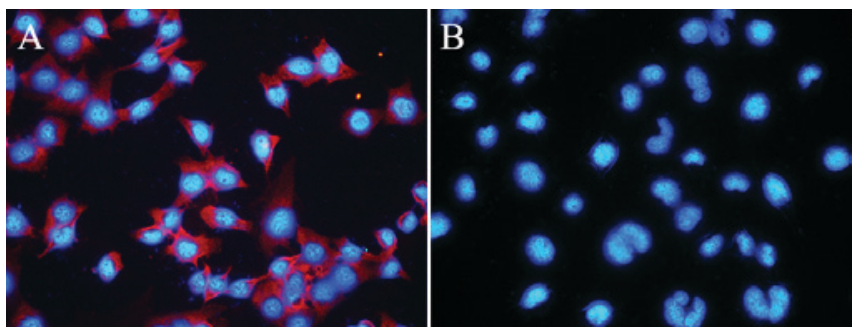

Figure 3. Imaging of EJ fixed cells labeled with (A) QD605-PSCA and (B) QD605. Magnification, x400. QD, quantum dot; PSCA, prostate stem cell antigen.

the action of DTT, the antibody fragments were reduced to expose thiol groups. The covalent bonding of antibodies onto QD605-SMCC occurred via the thiol and maleic imide groups. Finally, QD605-PSCA was obtained. The optical properties revealed that QD605-PSCA fluorescent probes retained the original basic optical characteristics of QD605, including a wide and continuous excitation spectrum, a narrow and symmetrical emission spectrum, good fluorescence stability and long fluorescence duration. The immune-fluorescence results revealed that the QD605-PSCA probes retained the PSCA protein immune activity and the PSCA was able to combine specifically with bladder cancer cell surface antigens. In addition, in the living cell labeling experiment, orange-red fluorescence was visible on the cell membrane and bright fluorescence was still visible after repeated laser irradiation. Over

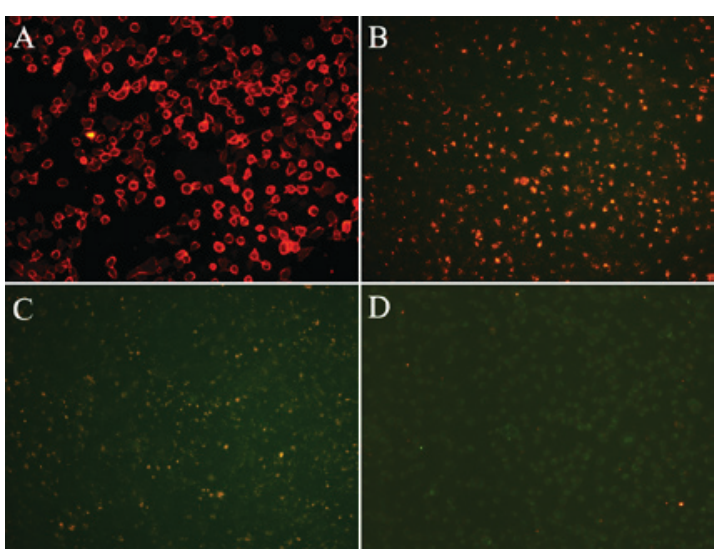

Figure 4. Fluorescence imaging of EJ living cells labeled with QD605-PSCA probes at (A) $30 \mathrm{~min}$, (B) $24 \mathrm{~h}$ and (C) $48 \mathrm{~h}$. (D) Fluorescence imaging of EJ living cells labeled with QD605. Magnification, x200. QD, quantum dot; PSCA, prostate stem cell antigen.

time, the fluorescence of QD605 appeared in the cytoplasm, which was considered to be indicative of cellular proliferation and endocytosis. The results of the present study demonstrate that QD605-PSCA probes are able to achieve targeted labeling and their fluorescence intensity is high and stable. The excellent optical properties and molecular targeting of QD-antibody probes may be useful for in vivo non-invasive targeted imaging and may contribute to early diagnosis, imaging localization and targeted therapies for tumors. 


\section{Acknowledgements}

The authors would like to thank D.W. Pang (College of Chemistry and Molecular Sciences and State Key Laboratory of Virology of Wuhan University) for his technical assistance in this study. The present study was performed at the Key Laboratory of Hubei Province for Digestive System Disease, Renmin Hospital of Wuhan University (Hubei, China).

\section{Funding}

The present study was supported by the National Natural Science Foundation of China (grant no. 81272826).

\section{Availability of data and materials}

The analyzed data sets generated during the present study are available from the corresponding author on reasonable request.

\section{Authors' contributions}

RY and TR were responsible for performing the experiments, collecting the data and writing the manuscript. FC, WY, YR, $\mathrm{XZ}$ and SL were responsible for the statistical analysis and manuscript revision. FC designed the study.

\section{Ethics approval and consent to participate}

Not applicable.

\section{Patient consent for publication}

Not applicable.

\section{Competing interests}

The authors declare that they have no competing interests.

\section{References}

1. Torre LA, Bray F, Siegel RL, Ferlay J, Lortet-Tieulent J and Jemal A: Global cancer statistic, 2012. CA Cancer J Clin 65: 87-108, 2015.

2. Siegel RL, Miller KD and Jemal A: Cancer statistics, 2015. CA Cancer J Clin 65: 5-29, 2015.

3. Chen W, Zheng R, Baade PD, Zhang S, Zeng H, Bray F, Jemal A $\mathrm{Yu}$ XQ and He J: Cancer statistics in china, 2015. CA Cancer J Clin 66: 115-132, 2016.

4. Sylvester RJ, van der Meijden AP, Oosterlinck W, Witjes JA, Bouffioux C, Denis L, Newling DW and Kurth K: Predicting recurrence and progression in individual patients with stage Ta T1 bladder cancer using EORTC risk tables: A combined analysis of 2596 patients from seven EORTC trials. Eur Urol 49: 466-475, 2006.

5. Resch-Genger U, Grabolle M, Cavaliere-Jaricot S, Nitschke R and Nann T: Quantum dots versus organic dyes as fluorescent labels. Nat Methods 5: 763-775, 2008.

6. Yu WW, Chang E, Drezek R and Colvin VL: Water-soluble quantum dots for biomedical applications. Biochem Biophys Res Commun 348: 781-786, 2006.

7. Smith AM, Ruan G, Rhyner MN and Nie S: Engineering luminescent quantum dots for in vivo molecular and cellular imaging. Ann Biomed Eng 34: 3-14, 2006.

8. Gonda K, Miyashita M, Higuchi $\mathrm{H}$, Tada H, Watanabe TM, Watanabe M, Ishida T and Ohuchi N: Predictive diagnosis of the risk of breast cancer recurrence after surgery by single-particle quantum dot imaging. Sci Rep 5: 14322, 2015.
9. Reiter RE, Gu Z, Watabe T, Thomas G, Szigeti K, Davis E, Wahl M, Nisitani S, Yamashiro J, Le Beau MM, et al: Prostate stem cell antigen: A cell surface marker overexpressed in prostate cancer. Proc Natl Acad Sci USA 95: 1735-1740, 1998.

10. Cunha AC, Weigle B, Kiessling A, Bachmann M and Rieber EP: Tissue-specificity of prostate specific antigens: Comparative analysis of transcript levels in prostate non-prostate tissues. Cancer Lett 236: 229-238, 2006

11. Amara N, Palapattu GS, Schrage M, Gu Z, Thomas GV, Dorey F, Said J and Reiter RE: Prostate stem cell antigen is overexpressed in human transitional cell carcinoma. Cancer Res 61: 4660-4665, 2001.

12. O'Toole CM, Povey S, Hepburn P and Franks LM: Identity of some human bladder cancer cell lines. Nature 301: 429-430, 1983.

13. Capes-Davis A, Theodosopoulos G, Atkin I, Drexler HG, Kohara A, MacLeod RA, Masters JR, Nakamura Y, Reid YA, Reddel RR and Freshney RI: Check your cultures! A list of cross-contaminated or misidentified cell lines. Int J Cancer 127: 1-8, 2010.

14. Lee PY, Costumbrado J, Hsu CY and Kim YH: Agarose gel electrophoresis for the separation of DNA fragment. J Vis Exp 20: e3923, 2012.

15. Barak Y, Meir I, Shapiro A, Jang Y and Lifshitz E: Fundamental properties in colloidal quantum dots. Adv Mater 19: e1801442, 2018.

16. Jung S and Chen X: Quantum dot-dye conjugates for biosensing, imaging, and therapy. Adv Healthc Mater 7: e1800252, 2018.

17. Matea CT, Mocan T, Tabaran F, Pop T, Mosteanu O, Puia C, Iancu C and Mocan L: Quantum dots in imaging, drug delivery and sensor applications. Int J Nanomedicine 12: 5421-5431, 2017.

18. Choi Y, Kim K, Hong S, Kim H, Kwon YJ and Song R: Intracellular protein target detection by quantum dots optimized for live cell imaging. Bioconjug Chem 22: 1576-1586, 2011.

19. Li H, Duan ZW, Xie P, Liu YR, Wang WC, Dou SX and Wang PY: Effects of paclitaxel on EGFR endocytic trafficking revealed using quantum dot tracking in single cells. PLoS One 7: e45465, 2012.

20. Allen PM, Liu W, Chauhan VP, Lee J, Ting AY, Fukumura D, Jain RK and Bawendi MG: InAs(ZnCdS) quantum dots optimized for biological imaging in the near-infrared. J Am Chem Soc 132: 470-471, 2010.

21. Cai W, Shin DW, Chen K, Gheysens O, Cao Q, Wang SX, Gambhir SS and Chen X: Peptide-labeled near-infrared quantum dots for Imaging tumor vasculature in living subjects. Nano Lett 6: 669-676, 2006.

22. Lu Z, Zhu Z, Zheng X, Qiao Y, Guo J and Li CM: Biocompatible fluorescence-enhanced $\mathrm{ZrO}_{2}$-CdTe quantum dot nanocomposite for in vitro cell imaging. Nanotechnology 22: 155604, 2011.

23. Rosenthal SJ, Chang JC, Kovtun O, McBride JR and Tomlinson ID: Biocompatible quantum dots for biological applications. Chem Biol 18: 10-24, 2011.

24. Goldman ER, Balighian ED, Mattoussi H, Kuno MK, Mauro JM, Tran PT and Anderson GP: Avidin: A natural bridge for quantum dot-antibody conjugates. J Am Chem Soc 124: 6378-6382, 2002.

25. Boeneman K, Deschamps JR, Buckhout-White S, Prasuhn DE, Blanco-Canosa JB, Dawson PE, Stewart MH, Susumu K, Goldman ER, Ancona M and Medintz IL: Quantum dot DNA bioconjugates: Attachment chemistry strongly influences the resulting composite architecture. ACS Nano 4: 7253-7266, 2010.

26. Akerman ME, Chan WC, Laakkonen P, Bhatia SN and Ruoslahti E: Nanocrystal targeting in vivo. Proc Natl Acad Sci USA 99: 12617-12621, 2002.

27. Daniels TR, Bernabeu E, Rodríguez JA, Patel S, Kozman M, Chiappetta DA, Holler E, Ljubimova JY, Helguera G and Penichet ML: The transferrin receptor and the targeted delivery of therapeutic agents against cancer. Biochim Biophys Acta 1820: 291-317, 2012.

28. Hermanson GT: Bioconjugate techniques. Academic Press, Amsterdam, 2013.

29. Susumu K, Oh E, Delehanty JB, Blanco-Canosa JB, Johnson BJ, Jain V, Hervey WJ IV, Algar WR, Boeneman K, Dawson PE and Medintz IL: Multifunctional compact zwitterionic ligands for preparing robust biocompatible semiconductor quantum dots and gold nanoparticles. J Am Chem Soc 133: 9480-9496, 2011.

30. Susumu K, Uyeda HT, Medintz IL, Pons T, Delehanty JB and Mattoussi H: Enhancing the stability and biological functionalities of quantum dots via compact multifunctional ligands. J Am Chem Soc 129: 13987-13996, 2007.

31. Li Z, Wang Y, Wang J, Tang Z, Pounds JG and Lin Y: Rapid and sensitive detection of protein biomarker using a portable fluorescence biosensor based on quantum dots and a lateral flow test strip. Anal Chem 82: 7008-7014, 2010. 